\title{
Comparison of gross tumor volume of primary oesophageal cancer based on contrast-enhanced three-dimensional, four- dimensional, and cone beam computed tomography
}

\author{
Chao-Yue Hu${ }^{1,2}$, Jian-Bin Li², Jin-Zhi Wang², Wei Wang², Feng-Xiang Li² and Yan- \\ Luan Guo ${ }^{2}$ \\ ${ }^{1}$ School of Medicine and Life Sciences, University of Jinan-Shandong Academy of Medical Sciences, Jinan, Shandong Province, \\ China \\ ${ }^{2}$ Department of Radiation Oncology, Shandong Cancer Hospital Affiliated to Shandong University, Shandong Academy of \\ Medical Sciences, Jinan, Shandong Province, China
}

Correspondence to: Jian-Bin Li, email: lijianbin@msn.com

Keywords: oesophageal cancer, three-dimensional computed tomography, four-dimensional computed tomography, cone beam computed tomography, gross tumor volume

Received: April 13, $2017 \quad$ Accepted: September 19, $2017 \quad$ Published: October 05, 2017

Copyright: Hu et al. This is an open-access article distributed under the terms of the Creative Commons Attribution License 3.0 (CC BY 3.0), which permits unrestricted use, distribution, and reproduction in any medium, provided the original author and source are credited.

\section{ABSTRACT}

Background: To explore motion information included in 3DCT, 4DCT and CBCT by comparing volumetric and positional differences of GTV.

Results: Independent of tumor location, significant differences were observed among volumes [IGTV $10>\left(\right.$ IGTV $_{\text {CBCT }}$ or IGTV $\left._{\text {MIP }}\right)>\left(G_{\text {TV }}\right.$ or GTV $\left.\left._{4 D 50}\right)\right]$. The underestimations or overestimations between IGTV I0 $_{10}$ and IGTV

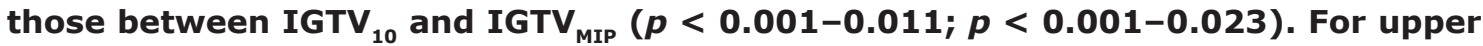
oesophageal tumors, $\mathrm{GTV}_{4 \mathrm{DS} 0} / \mathrm{IGTV}_{\mathrm{CBCT}}$ negatively correlated with motion vector ( $r$ $=-0.756, p=0.011)$. In AP direction, the centroid coordinates of IGTV from GTV ${ }_{3 D}$, GTV $_{4 D 50}$, IGTV $_{\text {MIP }}$ and IGTV ${ }_{10}(p=0.006,0.013,0.038$, and 0.010$)$. For middle oesophageal tumors, IGTV $_{10} /$ IGTV $_{\text {СвСт }}$ positively correlated with motion vector $(r=0.695, p=0.006)$. The centroid coordinates of IGTV ${ }_{\mathrm{CBCT}}$ differed from those of $\operatorname{IGTV}_{10}(p=0.046)$ in AP direction. For distal oesophageal tumors, the centroid coordinates of IGTV СBCT $_{\text {showed significant differences to those of IGTV }}$ MIP $(p=0.042)$ in LR direction. For both middle and distal tumors, the degrees of

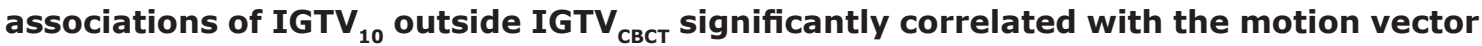
$(r=0.540, p=0.046 ; r=0.678, p=0.031)$.

Materials and Methods: Thirty-four oesophageal cancer patients underwent 3DCT, 4DCT and CBCT. GTV ${ }_{3 D}$, GTV $_{4 D 50}$, internal GTV ${ }_{\text {MIP }}\left(\right.$ IGTV $\left._{\text {MIP }}\right)$ and IGTV CBCT were delineated on 3DCT, $4 \mathrm{DCT}_{50}{ }{ }^{4 D C T}$ MIP and CBCT. GTVs from 10 respiratory phases were combined to produce $\mathrm{GTV}_{10}$. Differences in volume, position for different targets, correlation between volume ratio and motion vector were evaluated. The motion vector was the spatial moving of the target centroid position.

Conclusions: IGTV GTV $_{4 D 50}$ for upper oesophageal tumors, but slightly less than IGTV $_{10}$ for middle and distal oesophageal tumors. IGTV ${ }_{\text {CBCT }}$ incorporated similar motion information to IGTV MIP: $^{\text {* }}$ However, motion information encompassed in CBCT and MIP cannot replace each other.

\section{INTRODUCTION}

Radiotherapy (RT) have been the mainstream technologies for the patients with inoperable oesophageal cancer. Different computed tomography (CT) images may be involved in the process of radiotherapy. Threedimensional CT (3DCT) is a common positioning technology. In our study, the 3DCT images also known as 
"fast-CT" images were acquired in a short time. Although they only display the tumor during a certain moment of the breathing cycle [1], the temporal images acquired during 3DCT scanning encompass partial respiration-induced tumor motion information [2].

Oesophageal mobility can arise from peristalsis, respiratory motions, and cardiac action, with the predominant source of motion being respiratory [3]. Improved localization accuracy of the tumor can reduce the effect of radiation on the surrounding normal tissues and improve the therapeutic ratio. However, respirationinduced tumor motion can make target localization challenging [4-6]. Four-dimensional computed tomography (4DCT) is able to subdivide the CT datas acquired in a respiration cycle into a series of timeresolved $3 \mathrm{D}$ data $[7,8]$. The advent of $4 \mathrm{DCT}$ has made the measure of respiration-induced motion of oesophageal mobility possible. Currently, during lung stereotactic body radiation therapy (SBRT), 4DCT is routinely incorporated into the treatment planning to include information regarding not only the range of tumor motion but also the different spatial tumor positions [9]. Regarding the definition of internal target volumes (ITV) according to the International Commission on Radiation Units and Measurements report 62, the internal gross tumor volume (IGTV) incorporating the intrafraction motion of the GTV has been adopted in many studies [2, 10]. In our present study, the relationships of IGTVs IGTV $_{10}$ combined from ten 4DCT phases and IGTV $_{\text {MIP }}$ and IGTV $_{\mathrm{CBCT}}$ ) were analysed to explore the motion information included in IGTVs.

Prior to therapy delivery, it is crucial to localize the target volume using online imaging. Currently, cone beam CT (CBCT) is widely used for target verification, treatment planning modification, and image guidance during the delivery of radiation $[11,12]$. In terms of online target verification and correction techniques, CBCT can show motion displacement of the GTV in three directions by fusing CBCT images with treatment planning CT scans. And CBCT scans are acquired over a period of several respiratory cycles providing motion information. Recent studies which mainly concentrated on lung tumors have gradually begun to give careful attention to the motion information obtained by CBCT [13-15]. However, comparisons of target volumes for oesophageal cancer are limited in the literatures, therefore it is essential to investigate the variations in motion information between the target volumes derived from 3DCT, 4DCT, and CBCT images for oesophageal tumors.

\section{RESULTS}

\section{Variations of volumes}

The means and standard deviations (SD) of the volumes of $\mathrm{GTV}_{3 \mathrm{D}}, \mathrm{GTV}_{4 \mathrm{D} 50}$, IGTV $\mathrm{IIP}_{\text {M }}, \mathrm{IGTV}_{10}$, and
IGTV $_{\text {СвСт }}$ are listed in Table 1. For upper, middle, and distal oesophageal tumors, the variation rule of the target volumes were completely consistent. The $\mathrm{IGTV}_{10}$ was larger than the $\operatorname{IGTV}_{\text {MIP }}$ and $\operatorname{IGTV}_{\text {СвСт }}(p<0.001$ for both), whereas no significant differences were observed

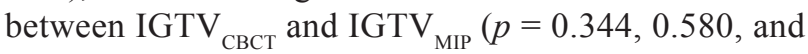
0.128 , respectively). The volumes of $\mathrm{IGTV}_{\mathrm{CBCT}}$ and IGTV $_{\text {MIP }}$ were also significantly larger than those of $\mathrm{GTV}_{3 \mathrm{D}}$ or $\mathrm{GTV}_{4 \mathrm{D} 50}(p<0.001-0.014)$, whereas there were no statistically significant differences between the $\mathrm{GTV}_{3 \mathrm{D}}$ and $\mathrm{GTV}_{4 \mathrm{D} 50}(p=0.691,0.187$, and 0.763, respectively).

The volume ratios of $\mathrm{GTV}_{3 \mathrm{D}}, \mathrm{GTV}_{4 \mathrm{D} 50}, \mathrm{IGTV}_{\text {MIP, }}$,

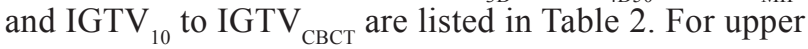
oesophageal tumors, the volume ratios of $\mathrm{GTV}_{4 \mathrm{D} 50}$ ' IGTV $_{\text {СвСт }}$ showed a significant correlation to the $3 \mathrm{D}$ direction motion vector $(\mathrm{r}=-0.756, p=0.011)$. However, GTV $_{3 \mathrm{D}} / \mathrm{IGTV}_{\mathrm{CBCT}}, \quad \mathrm{IGTV}_{\mathrm{MIP}} / \mathrm{IGTV}_{\mathrm{CBCT}}$, and $\mathrm{IGTV}_{10} / \mathrm{IGTV}_{\mathrm{CBCT}}$ demonstrated no significant correlations to the $3 \mathrm{D}$ direction motion vector $(p=$ 0.073-0.439). For middle oesophageal tumors, IGTV $10 /$ IGTV $_{\mathrm{CBCT}}$ showed a positive significant correlation to the 3D direction motion vector $(\mathrm{r}=0.695, p=0.006)$, whereas $\mathrm{GTV}_{3 \mathrm{D}} / \mathrm{IGTV}_{\mathrm{CBCT}}, \mathrm{GTV}_{4 \mathrm{D} 50} / \mathrm{IGTV}_{\mathrm{CBCT}}$, and $\mathrm{IGTV}_{\mathrm{MIP}} / \mathrm{IGTV}_{\mathrm{CBCT}} \operatorname{did} \operatorname{not}(p=0.091-0.762)$. For distal oesophageal tumors, no significant correlations were observed between the 3D direction motion vector and the volume ratios of $\mathrm{GTV}_{3 \mathrm{D}}, \mathrm{GTV}_{4 \mathrm{D} 50}, \mathrm{IGTV}_{\mathrm{MIP}}$, and $\mathrm{IGTV}_{10}$ to $\operatorname{IGTV}_{\mathrm{CBCT}}(p=0.131-0.921)$.

\section{Variations of the target centroid position}

The COM coordinates for all GTV or IGTV could be obtained through the TPS. For upper oesophageal tumors, in the AP direction, the centroid coordinates of IGTV $_{\mathrm{CBCT}}$ showed significant differences compared to those of $\mathrm{GTV}_{3 \mathrm{D}}, \mathrm{GTV}_{4 \mathrm{D} 50}, \mathrm{IGTV}_{\mathrm{MIP}}$, and $\mathrm{IGTV}_{10}$ ( $p=0.006,0.013,0.038$, and 0.010 , respectively), whereas the target centroid positions at other directions showed no significant differences. For tumors located in the middle thoracic oesophagus, in the AP direction, there were significant differences of the centroid coordinates between $\operatorname{IGTV}_{\mathrm{CBCT}}$ and $\operatorname{GTV}_{3 \mathrm{D}}(p=0.008)$, and between IGTV $_{\mathrm{CBCT}}$ and IGTV $\operatorname{IV}_{10}(p=0.046)$. Further, a significant difference was observed between the centroid position of IGTV $_{\mathrm{CBCT}}$ and $\operatorname{GTV}_{4 \mathrm{D} 50}(p=0.027)$ in the CC direction. For distal oesophageal tumors, variations in the centroid shift of IGTV СBCT $_{\text {and GTV }}$, IGTV IBCT $_{\text {CB }}$ and GTV $_{4 \mathrm{D} 50}$, and IGTV CBCT $_{\text {and IGTV }}$ MIP were found only in the LR direction ( $p=0.024,0.017$, and 0.042 , respectively).

\section{Differences in the inclusion relation}

The mean volumetric overestimations or underestimations for $\mathrm{GTV}_{3 \mathrm{D}}, \mathrm{IGTV}_{\mathrm{MIP}}$, and $\mathrm{IGTV}_{\mathrm{CBCT}}$ compared to the IGTV ${ }_{10}$ are listed in Table 3 . Irrespective 


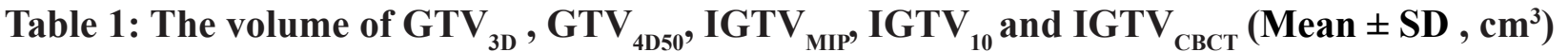

\begin{tabular}{lccccc}
\hline volume & $\mathbf{G T V}_{\text {3D }}$ & $\mathbf{G T V}_{\text {4D } 50}$ & IGTV $_{\text {MIP }}$ & IGTV $_{10}$ & IGTV $_{\text {CBCT }}$ \\
\hline upper & $11.11 \pm 2.86$ & $11.18 \pm 2.75$ & $12.63 \pm 2.93$ & $13.80 \pm 3.20$ & $12.77 \pm 3.12$ \\
middle & $27.30 \pm 12.46$ & $27.74 \pm 12.94$ & $29.22 \pm 12.94$ & $33.52 \pm 14.75$ & $29.40 \pm 12.70$ \\
distal & $21.69 \pm 11.88$ & $21.78 \pm 11.93$ & $22.97 \pm 12.17$ & $27.86 \pm 13.62$ & $23.89 \pm 11.99$ \\
\hline
\end{tabular}

Table 2: The volume ratios of $\mathrm{GTV}_{3 \mathrm{D}}, \mathrm{GTV}_{4 \mathrm{D} 50}$, IGTV ${ }_{\text {MIP }}$ and IGTV $_{10}$ to $\mathrm{IGTV}_{\mathrm{CBCT}}(\mathrm{Mean} \pm \mathrm{SD})$

\begin{tabular}{lcccc}
\hline & $\mathbf{G T V}_{\text {30 }} / \mathbf{I G T V}_{\mathbf{C B C T}}$ & $\mathbf{G T V}_{\text {4D50 }} / \mathbf{I G T V}_{\mathbf{C B C T}}$ & $\mathbf{I G T V}_{\mathbf{M I P}} / \mathbf{I G T V}_{\mathbf{C B C T}}$ & $\mathbf{I G T V}_{\mathbf{1 0}} / \mathbf{I G T V}_{\mathbf{C B C T}}$ \\
\hline upper & $0.87 \pm 0.03$ & $0.88 \pm 0.05$ & $0.99 \pm 0.04$ & $1.08 \pm 0.03$ \\
middle & $0.92 \pm 0.04$ & $0.93 \pm 0.05$ & $0.99 \pm 0.04$ & $1.14 \pm 0.07$ \\
distal & $0.87 \pm 0.11$ & $0.89 \pm 0.09$ & $0.94 \pm 0.10$ & $1.18 \pm 0.07$ \\
\hline
\end{tabular}

Table 3: The volume characteristics for GTV $_{3 \mathrm{D}}, \mathrm{IGTV}_{\mathrm{MIP}}$ and IGTV $\mathrm{CBCT}_{\text {compared with IGTV }}$ $($ Mean \pm SD, \%)

\begin{tabular}{|c|c|c|c|c|c|c|}
\hline & $\begin{array}{c}\text { GTV }_{3 \mathrm{D}} \text { outside } \\
\text { IGTV }_{10}\end{array}$ & $\begin{array}{c}\text { IGTV }_{10} \text { outside } \\
\text { GTV }_{3 \mathrm{D}}\end{array}$ & $\begin{array}{c}\text { IGTV }_{\text {MIP }} \\
\text { outside IGTV }\end{array}$ & $\begin{array}{l}\text { IGTV }_{10} \text { outside } \\
\text { IGTV }_{\text {MIP }}\end{array}$ & $\begin{array}{c}\text { IGTV }_{\text {СBCT }} \\
\text { outside IGTV } \\
\text { IGT }_{10}\end{array}$ & $\begin{array}{l}\text { IGTV }_{10} \text { outside } \\
\text { IGTV }_{\text {СвСт }}\end{array}$ \\
\hline upper & $2.80 \pm 1.69$ & $22.10 \pm 3.64$ & $5.20 \pm 3.01$ & $13.10 \pm 2.69$ & $16.00 \pm 4.35$ & $22.20 \pm 3.82$ \\
\hline middle & $3.36 \pm 2.90$ & $21.60 \pm 5.24$ & $2.43 \pm 2.06$ & $14.79 \pm 3.49$ & $11.93 \pm 4$ & $22.14 \pm 2.63$ \\
\hline distal & $6.20 \pm 7.65$ & $29.70 \pm 12.27$ & $1.20 \pm 0.42$ & $20.70 \pm 7.51$ & $14.20 \pm 6.41$ & $26.90 \pm 5.92$ \\
\hline
\end{tabular}

Abbreviations: $\mathrm{GTV}_{3 \mathrm{D}}$, the gross tumor volumes of three-dimensional computed tomography; $\mathrm{GTV}_{4 \mathrm{D} 50}$, the gross tumor volumes of the end expiration phase of four-dimensional computed tomography; IGTV $_{\text {MIP }}$, the internal gross tumor volumes of the maximum intensity projection of four-dimensional computed tomography; $\mathrm{IGTV}_{10}$, the union of gross tumor volumes from all ten phase images of four-dimensional computed tomography; IGTV $_{\mathrm{CBCT}}$, the internal gross tumor volumes of cone beam computed tomography.

of the tumor location, the volumetric underestimation percentages of $\mathrm{IGTV}_{10}$ outside $\mathrm{IGTV}_{\mathrm{CBCT}}$ were larger than those of $\operatorname{IGTV}_{10}$ outside $\operatorname{IGTV}_{\text {MIP }}(p<0.001-0.011)$, whereas no significant differences were observed between IGTV $_{10}$ outside $\operatorname{IGTV}_{\text {CBCT }}$ and $\operatorname{IGTV}_{10}$ outside $\operatorname{GTV}_{3 \mathrm{D}}(p$ $=0.357-0.943)$. The mean volumetric overestimations for IGTV $_{\mathrm{CBCT}}$ compared with IGTV 10 were larger than those of $\operatorname{IGTV}_{\mathrm{MIP}}$ compared with $\operatorname{IGTV}_{10}(p<0.001-0.023)$. Similar results were found between IGTV $\mathrm{GTV}_{3 \mathrm{D}}$ outside $\operatorname{IGTV}_{10}(p<0.001)$. For tumors located in the middle and distal thoracic oesophagus, the degrees of associations of IGTV $_{10}$ outside IGTV significant correlations to the $3 \mathrm{D}$ direction motion vector $(\mathrm{r}=0.540, p=0.046 ; \mathrm{r}=0.678, p=0.031)$.

\section{Differences in the MI}

The mean values of the MIs between $\mathrm{GTV}_{3 \mathrm{D}}$ and

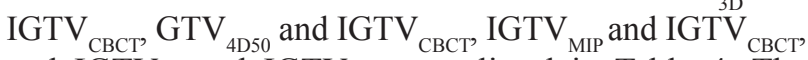
and $\mathrm{IGTV}_{10}$ and $\mathrm{IGTV}_{\text {Свст }}$ are listed in Table 4. The mean MIs ranged from 0.65 to 0.72 . The MI values of IGTV $_{\text {MIP }}$ relative to IGTV upper, middle, and distal thoracic oesophageal tumors $(p=0.235-0.863)$.

\section{DISCUSSION}

Target volume definition is a crucial step during the process of precise radiotherapy. The key to delineate GTV of oesophageal tumor is to define the boundaries of the primary tumor. The boundaries between the oesophagus and peripheral tissues such as the ventricles or descending aorta may be blurred on non-contrast enhanced CT images. Consequently, those problem will cause inaccuracies in the GTV delineation or IGTV construction. However, CE-CT scans can enhance the tumor margin between peripheral tissues, making it easy to determine the range of the tumor. Theoretically, in our study, intravenous contrast agents were used to acquired $\mathrm{CE}-\mathrm{CT}$ images to improve the margin contrast between tissues and reduce the delineation uncertainty. Few studies have focused on the comparison of target volumes for oesophageal cancer using different CT imaging modalities. Therefore, we investigate the variations in 


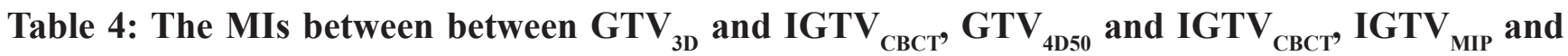
IGTV $_{\mathrm{CBCT}}$, and IGTV 10 and IGTV IBCT $_{10}($ Mean \pm SD)

\begin{tabular}{lcccc}
\hline & $\mathbf{G T V}_{3 \mathrm{D}}-\mathbf{I G T V}_{\mathrm{CBCT}}$ & $\mathbf{G T V 4}_{\mathrm{D} 50} \mathbf{I G T V}_{\mathrm{CBCT}}$ & $\mathbf{I G T V}_{\mathrm{MIP}}-\mathbf{I G T V}_{\mathrm{CBCT}}$ & $\mathbf{I G T V}_{10}-\mathbf{I G T V}_{\mathrm{CBCT}}$ \\
\hline upper & $0.68 \pm 0.06$ & $0.65 \pm 0.05$ & $0.69 \pm 0.06$ & $0.68 \pm 0.06$ \\
middle & $0.72 \pm 0.05$ & $0.68 \pm 0.04$ & $0.71 \pm 0.03$ & $0.70 \pm 0.03$ \\
distal & $0.68 \pm 0.06$ & $0.67 \pm 0.07$ & $0.66 \pm 0.09$ & $0.65 \pm 0.08$ \\
\hline
\end{tabular}

Abbreviations: $\mathrm{GTV}_{3 \mathrm{D}}$, the gross tumor volumes of three-dimensional computed tomography; $\mathrm{GTV}_{4 \mathrm{D} 50}$, the gross tumor volumes of the end expiration phase of four-dimensional computed tomography; IGTV $_{\text {MIP }}$, the internal gross tumor volumes of the maximum intensity projection of four-dimensional computed tomography; $\mathrm{IGTV}_{10}$, the union of gross tumor volumes from all ten phase images of four-dimensional computed tomography; $\mathrm{IGTV}_{\mathrm{CBCT}}$, the internal gross tumor volumes of cone beam computed tomography.

motion information among the target volumes derived from 3DCT, 4DCT, and CBCT images for oesophageal tumors.

4DCT images, which is a novel method of portraying tumor motion, can provide the measure of breathing-induced tumor motion of the internal anatomy of the patient [16]. Previous studies considered that the end expiration phase was the most stable phase [17, 18], and $\mathrm{GTV}_{4 \mathrm{D} 50}$ has been considered the closest measurement to the actual tumor size $[19,20]$. Accordingly, the motion information encompassed in $\mathrm{GTV}_{4 \mathrm{D} 50}$ is considered the lowest. In our data, we demonstrated that $\mathrm{GTV}_{4 \mathrm{D} 50} / \mathrm{IGTV}_{\mathrm{CBCT}}$ showed a significant inverse correlation to motion vector for upper oesophageal tumors, indicating an increase in the variation of the $\mathrm{IGTV}_{\mathrm{CBCT}}$ size with the increasing tumor motion amplitude. This might be due to the $\mathrm{GTV}_{4 \mathrm{D} 50}$ was considered the closest measurement to the actual tumor size, and would thus reflect the actual oesophageal tumor size [20]. In addition, oesophageal mobility can arise from peristalsis and respiratory and cardiac actions. The upper oesophagus passes through the sternal notch and above the left atrium, along with numerous other structures, and is therefore generally very well fixed. Hence, the upper oesophagus has been reported to be the least affected by cardiac action (left atrium) and respiratory motion, with the lowest motion amplitudes $(0.16,0.14$, and $0.29 \mathrm{~cm}$ in the LR, AP, and CC directions, respectively) [10]. Consequently, $\mathrm{GTV}_{4 \mathrm{D} 50}$ could reflect the actual oesophageal tumor size. However, the CBCT scans are acquired over a period of several respiratory cycles providing sufficient information on respiration motion. Therefore, the motion information encompassed in the IGTV $_{\text {CBCT }}$ can be accurately reflected by comparing the variation in volume sizes between $\mathrm{IGTV}_{\mathrm{CBCT}}$ and $\mathrm{GTV}_{4 \mathrm{D} 50}$. In this study, the volume ratio correlated well with the motion vector, suggesting that $\mathrm{IGTV}_{\mathrm{CBCT}}$ incorporates much more motion information than $\mathrm{GTV}_{4 \mathrm{D} 50}$ for upper oesophageal tumors.

At present, CE-3DCT was widely used for the positioning of oesophageal cancer as most patients can tolerate it. Generally speaking, margins derived from population-based or site-specific tumor motion information of 4DCT are used to guide the expansion from GTV based on 3DCT to IGTV. However, the crucial premise is that 3DCT does not encompass much more motion information than the GTV from a single phase. Herein, we demonstrated that the ratios of $\mathrm{GTV}_{3 \mathrm{D}} / \mathrm{GTV}_{4 \mathrm{D} 50}$ for the upper, middle, and distal oesophagus approached 1, similar to what has been reported for lung cancer $[2,20]$. This result suggests that $\mathrm{GTV}_{3 \mathrm{D}}$ encompasses similar motion information as $\mathrm{GTV}_{4 \mathrm{D} 50}$. Consequently, we considered that IGTV $_{\mathrm{CBCT}}$ incorporates much more respiration motion information than $\mathrm{GTV}_{3 \mathrm{D}}$. Given that the centroid coordinates between $\mathrm{GTV}_{4 \mathrm{D} 50}$ and $\mathrm{GTV}_{3 \mathrm{D}}$ showed no significant difference in any of the three directions ( $p=0.204,0.149$, and 0.505 , respectively), and the relative high inclusion relations of $\mathrm{GTV}_{3 \mathrm{D}}$ in $\mathrm{GTV}_{4 \mathrm{D} 50}$ and $\mathrm{GTV}_{4 \mathrm{D} 50}$ in $\mathrm{GTV}_{3 \mathrm{D}}(0.88$ and 0.87 , respectively), which demonstrated no significant difference for the whole group $(\mathrm{t}=1.525, p=0.137)$, 3DCT may be regarded as an alternative when 4DCT images are not or cannot be acquired.

During the course radiotherapy, 3DCT simulation scans were routinely used for patient's positioning, and $\mathrm{CBCT}$ were widely used for target verification and image guidance during the delivery of radiation. As technology advances, the image resolution and scanning range of CBCT has improved. The applications of $\mathrm{CBCT}$ are further explored. 3DCT scanning repositioning are usually needful to define the patient's position repeatability and the necessity of modifying the target during the course of radiotherapy. So we explore the matching of the motion information included in 3DCT and CBCT to realize whether we could utilize CBCT instead of 3DCT to modify the treatment planning. Whether we could delineate IGTV $_{\text {СBCT }}$ on CBCT images acquired after times of radiotherapy, and then map IGTV $_{\text {СвСт }}$ to first positioned 3DCT directly to modify the treatment planning without the course of 3DCT scanning repositioning, provided that: a) the two target volumes match very well delineated on CBCT images acquired at the first treatment fraction and first positioned 3DCT 
images; b) the patient's position repeatability was very perfect during the course of radiotherapy; c) the tumor was obviously shrinked during the course of radiotherapy. We analysed the inclusion relations between $\mathrm{GTV}_{3 \mathrm{D}}$ and IGTV $_{10}$, and between IGTV been reported to provide the best overall representation of the 'true' moving GTV and incorporates the whole tumor respiration motion information envelope throughout the entire breathing cycle, though its delineation is the most time-consuming $[10,21,22]$. Therefore, we regarded IGTV $_{10}$ as the reference to reflect the differences in motion information contained in the target volumes on different CT modalities. However, in the part of results we found that the proportion of the volumetric overestimations for IGTV $_{\mathrm{CBCT}}$ outside $\mathrm{IGTV}_{10}$ were larger than those for $\mathrm{GTV}_{3 \mathrm{D}}$ outside $\mathrm{IGTV}_{10}$. The result did not meet the first premise. Therefore, 3DCT simulation scans are needed for repositioning during the course of radiotherapy.

From the results of the present study, we found no significant difference among the $\mathrm{IGTV}_{\mathrm{MIP}} / \mathrm{IGTV}_{\mathrm{CBCT}}$ for upper, middle, and distal oesophageal tumors $(\mathrm{F}=2.043, p=0.147)$. Meanwhile, no good correlation was observed between the motion vector and $\mathrm{IGTV}_{\mathrm{MIP}} /$ IGTV $_{\mathrm{CBCT}}$, irrespective of where the tumor was located $(\mathrm{r}$ $=-0.301, p=0.398 ; \mathrm{r}=0.458, p=0.099$; and $\mathrm{r}=-0.113$, $p=0.755$, respectively). These results indicated that the tumor motion amplitude had no effect on the variation in $\mathrm{IGTV}_{\mathrm{MIP}} / \mathrm{IGTV}_{\mathrm{CBCT}}$ and that the changes in $\mathrm{IGTV}_{\mathrm{CBCT}}$ and IGTV $_{\text {MIP }}$ may have a similar magnitude. Therefore, this suggests that the motion information encompassed in IGTV $_{\text {CBCT }}$ was parallel to that of IGTV MIP.

Independent of the tumor location, the percentages of volumetric underestimations or overestimations between IGTV $_{10}$ and IGTV

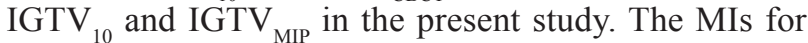
IGTV $_{\text {MIP }}$ relative to IGTV 0.66 for upper, middle and distal thoracic oesophageal tumors, respectively. The main cause of this phenomenon may be the variations in the target centroid position

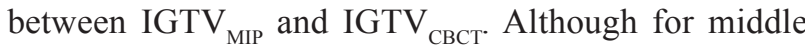
and distal oesophageal tumors, the predominant source of motion is respiratory [3]. As a tube-shaped and nonrigid organ in the mediastinum, in addition to peristalsis and respiration-induced movement, motion of the heart and aorta resulting from the cardiac cycle can also result in motion and volume deformation of the oesophagus [3, 23]. Accordingly, respiration-induced movement, and the squashing and stretching of the heart to the esophageal tumors might be factors responsible for the differences in the COM between IGTV $_{\text {MIP }}$ and IGTV $_{\text {СBCT }}$ in the AP and LR directions. Especially, for middle and distal oesophageal cancer, the effects of the variations in the position and volume of the heart on oesophageal targets cannot be ignored [24]. Of note, similar findings have been observed in lung cancer, as Seppenwoold et al. [25] reported that the target was affected by cardiac action more obviously in the LR direction when the lung tumors were located near the heart. Thus, IGTV $_{\mathrm{CBCT}}$ and $\mathrm{IGTV}_{\mathrm{MIP}}$ encompass similar respiration motion information; however, due to the spatial mismatch of $\mathrm{IGTV}_{\mathrm{CBCT}}$ and IGTV $_{\text {MIP }}$, the target motion information encompassed in CBCT and MIP images cannot replace each other.

The present study showed that the volume of IGTV $_{10}$ was larger than IGTV that $\mathrm{IGTV}_{10} / \mathrm{IGTV}_{\text {СBCT }}$ correlated well with the motion vector. These findings demonstrate that the change in $\mathrm{IGTV}_{10}$ was more obvious than the change in $\mathrm{IGTV}_{\mathrm{CBCT}}$ along with the increase in the tumor motion amplitude. In this study, the volume ratio correlated well with the motion vector, suggesting that $\mathrm{IGTV}_{10}$ incorporates much more motion information than $\mathrm{IGTV}_{\mathrm{CBCT}}$. When comparing IGTV sizes, we found that if IGTV 10 was chosen as the reference for the standard volume, $22.20 \%, 22.14 \%$, and $26.90 \%$ of $\mathrm{IGTV}_{10}$ volume tumor tissues would not receive irradiation, whereas $16.00 \%, 11.93 \%$, and $14.20 \%$ of the volume IGTV $_{\text {СВCT }}$ normal tissues would be inevitably irradiated for upper, middle, and distal oesophageal tumors, respectively. Furthermore, our study showed the correlation of $\operatorname{IGTV}_{10}$ outside IGTV $_{\text {СвСт }}$ and the spatial motion vector. This result showed the proportion of IGTV I0 $_{10}$ outside IGTV motion increased. It indicated that the larger the motion amplitude of the tumors, the more motion information of IGTV $_{\text {СBCT }}$ would be missed compared to IGTV I0 $_{10}$ The MI and inclusion relation reflect the translation, deformation, volume change, or rotation of the two selected volumes, which in turn affect the volumetric size, shape, and spatial position [26]. The variations of the target centroid position between IGTV $_{10}$ and IGTV the volumetric mismatch.

For upper oesophageal tumors, in the AP direction, the centroid coordinates of $\mathrm{IGTV}_{\mathrm{CBCT}}$ differed from those of IGTV $_{10}$ in the present study. As the upper oesophagus is generally very well fixed by numerous adjacent structures, it is the least affected by cardiac action and respiratory motion, with the least motion amplitude. Oesophageal mobility can arise from peristalsis, and attention should be paid to the day-to-day oesophageal peristalsis [12], as oesophageal intrafractional tumor position variation during irradiation delivery is one of the geometrical uncertainties that may affect the target centroid position. Oesophageal peristalsis in the AP direction might contribute to spatial position differences, which consequently leads to the differences in the quantity of motion information incorporated in $\mathrm{IGTV}_{\mathrm{CBCT}}$ and $\mathrm{IGTV}_{10}$. Furthermore, setup errors might have also contributed to the observed difference. Yamashita et al. [12] reported that the setup error was $4 \mathrm{~mm}$ (maximum, $11 \mathrm{~mm}$ ) in the longitudinal direction, $2 \mathrm{~mm}$ (maximum, $8 \mathrm{~mm}$ ) in the lateral direction, and $4 \mathrm{~mm}$ (maximum, $13 \mathrm{~mm}$ ) in the vertical direction. 
Other factors that likely contributed to the differences in spatial mismatch and volume size between the two target volumes should also be highlighted. First, free breathing CBCT image reconstruction makes use of every pixel in each projection, resulting in an averaging of the CT numbers across the image set. Accordingly, this image is meant to represent the time-averaged position of the target and results in a CT number averaging effect $[15,27]$. However, 4DCT images were a series of timeresolved $3 \mathrm{D}$ data. Due to the different image principle of 4DCT and CBCT, it may be one factor to lead the difference of the volume size and volumetric mismatch between two target volumes on different CT images. In addition, variations in the breathing pattern may be another potential reason for the observed differences during the acquisition of 3DCT, 4DCT, and CBCT scans. Clements et al. [15] reported that the sinusoidal patterns represented the ideal clinical scenario. However, in the present study, all CT image acquisitions were acquired during free breathing, without any breathing control, and this is bound to the variations of the target centroid position and volume size, as well as to the low MI between the two selected volumes.

There were some limitations in the present study. Most importantly, it should be noted that intraobserver target delineation error might reduce the accuracy of GTVs delineated on distinct patterns of CT images. To minimize systematic delineation uncertainty, the contouring was performed by a single physician with more than 5 years of experience using a unified standard, despite the fact that contouring the target volume on 10 different phases increases the workload and is time-consuming. Additionally, it is impossible to avoid the impact of registration errors. Boswell et al. [28] reported that the repositioning accuracy of automatic registration in a helical tomotherapy system was sub-millimetre. However, errors in alignment and patient setup displacements still exist, and it is possible that such errors, albeit minor, may have caused variations in the target centroid position and resulted in the low matching between the two target volumes. Nevertheless, despite the limitations in this study, our results can reflect the amount of respiration motion information encompassed in different CT modalities.

\section{MATERIALS AND METHODS}

\section{Patient selection and characteristics}

Thirty-four patients (19 men and 15 women) with pathologically confirmed oesophageal cancer, scheduled to undergo radiotherapy with 3D-CRT or IMRT, were enrolled between August 2014 and December 2015. The median age was 71 years (range, $41-83$ years). Among the 34 patients, 33 and 1 were diagnosed with squamous cell carcinoma and undifferentiated carcinoma, respectively.
The primary tumors were located in the upper, middle, and distal oesophagus in 10,14 , and 10 patients, respectively, based on the 7 th edition of Esophageal Cancer Staging published in 2009 from the American Joint Committee on Cancer (AJCC) and Union for International Cancer Control (UICC). No patient had been previously treated with thoracic radiotherapy and no patient had poor pulmonary function.

Written informed consent was obtained from all patients before the treatment was initiated. The study was approved by the Institutional Review Board (Shandong Cancer Hospital Affiliated to Shandong University Ethics Committee).

\section{CT data acquisition}

All patients were in the vacuum bags while being scanned. After laser alignment, contrast enhanced (CE)-3DCT and CE-4DCT were performed during free breathing using a 16-slice CT scanner (Philips Brilliance Bores CT Inc., Cleveland, $\mathrm{OH}$, USA). The standard acquisition parameters of the CE-3DCT were $120 \mathrm{kV}$ and $200 \mathrm{~mA}$. A total of $45 \mathrm{~mL}$ of iodinated contrast medium were infused at a rate of $1.5 \mathrm{~mL} / \mathrm{s}$. The CE-3DCT scans were produced per gantry rotation $(1 \mathrm{~s})$ and interval $(1.8 \mathrm{~s})$ between rotations. The slice thickness of the 3DCT scan was $3 \mathrm{~mm}$. When the CE-3DCT was finished, the CE-4DCT scan was sequentially initiated. The standard acquisition parameters of the CE-4DCT were $120 \mathrm{kV}$ and $400 \mathrm{~mA}$. A total of $55 \mathrm{~mL}$ of iodinated contrast medium were infused continuously at a rate of $1.0 \mathrm{~mL} / \mathrm{s}$. The CE-4DCT scan was acquired in helical mode with the scanning pitch ranging between 0.09 and 0.15 . During the CE-4DCT image acquisition, the respiratory signal was recorded with the Varian realtime position management (RPM) system (Varian Medical Systems, Palo Alto, CA, USA) by tracking the trajectory of the infrared markers placed on the epigastric region of the patient's abdomen. The resultant signal was sent to the scanner to label a time tag on each image. GE Advantage 4D software (GE Healthcare, Waukesha, WI, USA) was used to sort the reconstructed 4DCT images into 10 respiratory phases according to the phase of the breathing signal based on these tags labelled as $0-90 \%$. Phase $0 \%$ denoted the maximum end inspiration, and phase 50\% denoted the maximum end expiration. The maximum intensity projection (MIP) images were created from the raw 4DCT data. In the MIP images, each pixel was assigned the highest density value that occurred, taking into account all 10 respiratory phases.

Prior to the first treatment fraction, CE-CBCT scans were acquired using a kilovoltage CBCT scanner (Varian Medical Systems, Palo Alto, CA, USA) with the patient in 
the treatment position. The patients were aligned according to the skin tattoos by using the in-room laser system. The standard acquisition parameters were $120 \mathrm{kV}$ and $1000 \mathrm{~mA}$. The iodinated contrast medium was infused at a rate of 1.8 $\mathrm{mL} / \mathrm{s}$. The scan time was approximately one minute. The Eclipse system included software using a mutual information algorithm for automatic registration from the CE-4DCT and CE-CBCT to CE-3DCT. Finally, all images were transferred to the Eclipse treatment planning system (TPS) (Eclipse 8.6; Varian Medical Systems, Palo Alto, CA) for structure delineation and treatment planning. In our study, the 3DCT images were used for target delineation and treatment planning. 4DCT images were used for target delineation. The CBCT was used for target delineation and verification.

\section{GTV delineation}

In all cases, the GTV and/or IGTV contouring were completed by the same clinician, with more than 5 years of experience performing target volume delineation, using the mediastinal window setting. The GTV and/or IGTV for each patient were delineated as follows: (1) the $\mathrm{GTV}_{3 \mathrm{D}}$ and GTV $\mathrm{GD}_{40}$ were delineated on CE-3DCT and $\mathrm{CE}-4 \mathrm{DCT}_{50}$ images (the end expiration phase of CE-

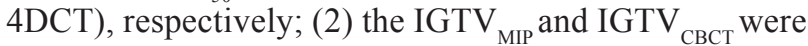
delineated separately on the CE-4DCT ${ }_{\text {MIP }}$ (the maximum intensity projection of CE-4DCT) and CE-CBCT datasets, respectively; and (3) the GTVs on each of the 10 respiratory phases of the CE-4DCT images were delineated and combined to produce $\operatorname{IGTV}_{10}($ Figure 1).

\section{Tumor motion}

The centre of mass (COM) coordinates represent the target centroid position. The target centroid position was reflected by the COM coordinates. The COM coordinates for all GTV or IGTV could be obtained through the TPS. The intrafractional displacements of the COM in the $x$ (left-right [LR]), $y$ (anterior-posterior [AP]), and $z$ (cranial- caudal [CC]) directions throughout 10 phases of 4DCT could also be obtained. The maximum tumor displacement in the LR, AP, and $\mathrm{CC}$ directions throughout 10 phases of $4 \mathrm{DCT}$ were calculated as $\Delta x, \Delta y$, and $\Delta z$, respectively. Subsequently, the 3D motion vector of the COM from the 4DCT was calculated according to the following formula: Vector $=\sqrt{\mathrm{D} x^{2}+\mathrm{D} y^{2}+\mathrm{D} z^{2}}$.

\section{Volume comparisons}

The volume, inclusion relation, and matching index (MI) were compared among the GTV $\left(\mathrm{GTV}_{3 \mathrm{D}}\right.$ and

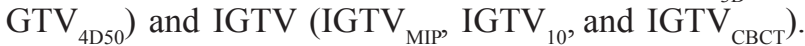
The percentage of A not included in B [Per (A not in B)] is used to indicate the inclusion relation between two volumes. The target A outside B was represented by Per (A not in B). Assumed volume B was reference for the standard volume irradiated. If the treatment planning was based on volume A, there would be Per (B not in A) of volume $\mathrm{B}$ missing irradiation which means the volumetric underestimation and Per (A not in B) of volume A being irradiated unnecessarily which means the volumetric overestimation. The formula is as follows: Per (A not in $\mathrm{B})=1-\mathrm{A} \cap \mathrm{B} / \mathrm{A}[21]$.

The MI was defined as the ratio of the intersection of volume $\mathrm{A}$ with volume $\mathrm{B}$ divided by the union of $\mathrm{A}$ and $\mathrm{B}$, as follows: $\mathrm{MI}=\mathrm{A} \cap \mathrm{B} / \mathrm{A} \cup \mathrm{B}$ [26]. The $\mathrm{MI}$ indicated translation, deformation, volume change, or rotation of the two selected volumes. The ideal value of the MI is 1; upon any change in size, shape, position, or orientation, the value of MI would be $<1$.

\section{Statistical analyses}

Statistical analyses were performed using SPSS software (version 19.0; SPSS Inc., Chicago, IL, USA). For all parameters, a normal distribution test (Q-Q plot) was performed. The paired sample t test was used for

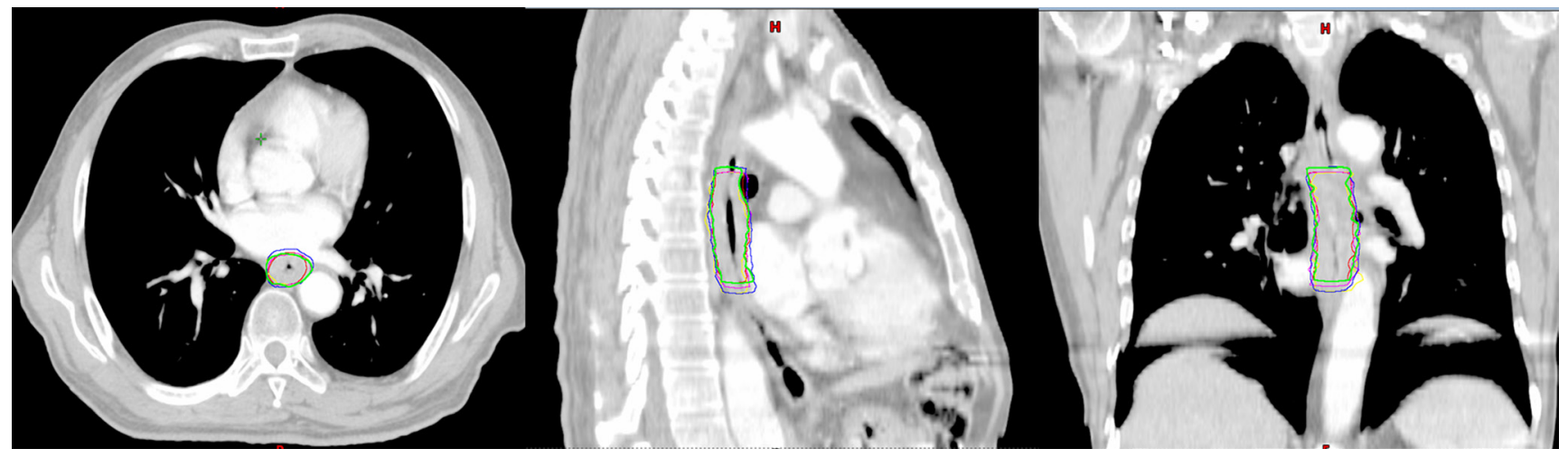

Figure 1: Gross target volume (GTV) or internal GTV (IGTV) formation in one patient: (green), GTV3D; (red),

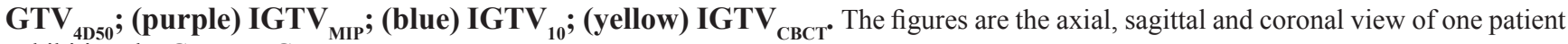
exhibiting the GTV or IGTVs. 
comparisons of tumor position, volumetric size, MI, and inclusion relation. The degree of associations between continuous variables and the 3D motion vector according to 4DCT was calculated using Pearson's test. For all analyses, a $p$ value $<0.05$ was regarded as significant.

\section{CONCLUSIONS}

Our findings indicate that the CBCT images incorporated much more respiration motion information than 3DCT images and the end expiration phase of 4DCT images for upper oesophageal tumors, but less than that of 10 respiratory phases of the 4DCT datasets for middle and distal oesophageal tumor. Simultaneously, CBCT images incorporated similar respiration motion information to MIP images. Nevertheless, the target motion information encompassed in CBCT and MIP images cannot replace each other.

\section{Author contributions}

CYH and JBL participated in the study design, contributed to the data collection, and draft the manuscript. WW made important contributions in delineating the oesophageal tumor. JZW and FXL made important contributions in revising the content. YLG contributed to data collection and analysis. All authors read and approved the final manuscript.

\section{CONFLICTS OF INTEREST}

The authors declare that they have no conflicts of interest.

\section{FUNDING}

This work was supported by National Key Research Program of China (2016YFC0904700), Science and Technology Plan Projects of Shandong Academy of Medical Sciences (Youth Fund: 2015-51) and Key Research and Development Program of Shandong Province (2016GSF201093).

\section{REFERENCES}

1. Hof H, Rhein B, Haering P, Kopp-Schneider A, Debus J, Herfarth K. 4D-CT-based target volume definition in stereotactic radiotherapy of lung tumors: comparison with a conventional technique using individual margins. Radiother Oncol. 2009; 93:419-23.

2. Li F, Li J, Zhang Y, Xu M, Shang D, Fan T, Liu T, Shao Q. Geometrical differences in gross target volumes between 3DCT and 4DCT imaging in radiotherapy for non-smallcell lung cancer. J Radiat Res. 2013; 54:950-6.
3. Hashimoto T, Shirato H, Kato M, Yamazaki K, Kyrauchi N, Morikawa T, Shimizu S, Ahn YC, Akine Y, Miyasaka K. Real-time monitoring of a digestive tract marker to reduce adverse effects of moving organs at risk (OAR) in radiotherapy for thoracic and abdominal tumors. Int $\mathrm{J}$ Radiat Oncol Biol Phys. 2005; 61:1559-64.

4. Takeda A, Kunieda E, Shigematsu N, Hossain DM, Kawase T, Ohashi T, Fukada J, Kawaguchi O, Uematsu M, Takeda T, Takemasa K, Takahashi T, Kubo A. Small lung tumors: long-scan-time CT for planning of hypofractionated stereotactic radiation therapy-initial findings. Radiology. 2005; 237:295-300.

5. Underberg RW, Lagerwaard FJ, Slotman BJ, Cuijpers JP, Senan S. Benefit of respiration-gated stereotactic radiotherapy for stage I lung cancer: an analysis of 4DCT datasets. Int J Radiat Oncol Biol Phys. 2005; 62:554-560.

6. O'Dell WG, Schell MC, Reynolds D, Okunieff R. Dose broadening due to target position variability during fractionated breath-held radiation therapy. Med Phys. 2002; 29:1430-1437.

7. Keall PJ, Starkschall G, Shukla H, Forster KM, Ortiz V, Stevens CW, Vedam SS, George R, Guerrero T, Mohan R. Acquiring 4D thoracic CT scans using a multislice helical method. Phys Med Biol. 2004; 49:2053-67.

8. Vedam SS, Keall PJ, Kini VR, Mostafavi H, Shukla HP, Mohan R. Acquiring a four-dimensional computed tomography dataset using an external respiratory signal. Phys Med Biol. 2003; 48:45-62.

9. Sonke JJ, Rossi M, Wolthaus J, van Herk MB, Damen E, Belderbos J. Frameless stereotactic body radiotherapy for lung cancer using four-dimensional cone beam CT guidance. Int J Radiat Oncol Biol Phys. 2009; 74:567-74.

10. Wang W, Li J, Zhang Y, Li F, Xu M, Fan T, Shao Q, Shang D. Comparison of patient-specific internal gross tumor volume for radiation treatment of primary esophageal cancer based separately on three-dimensional and four-dimensional computed tomography images. Dis Esophagus. 2014; 27:348-54.

11. Hawkins MA, Aitken A, Hansen VN, McNair HA, Tait DM. Set-up errors in radiotherapy for oesophageal cancers is electronic portal imaging or conebeam more accurate? Radiother Oncol. 2011; 98:249-54.

12. Yamashita H, Haga A, Hayakawa Y, Okuma K, Yoda K, Okano Y, Tanaka K, Imae T, Ohtomo K, Nakagawa K. Patient setup error and day-to-day esophageal motion error analyzed by cone-beam computed tomography in radiation therapy. Acta Oncol. 2010; 49:485-90.

13. Wang Z, Wu QJ, Marks LB, Larrier N, Yin FF. Cone-beam CT localization of internal target volumes for stereotactic body radiotherapy of lung lesions. Int J Radiat Oncol Biol Phys. 2007; 69:1618-24.

14. Liu HW, Khan R, D'Ambrosi R, Krobutschek K, Nugent Z, $\mathrm{Lau} \mathrm{H}$. The influence of target and patient characteristics on 
the volume obtained from cone beam CT in lung stereotactic body radiation therapy. Radiother Oncol. 2013; 106:312-6.

15. Clements N, Kron T, Franich R, Dunn L, Roxby P, Aarons Y, Chesson B, Siva S, Duplan D, Ball D. The effect of irregular breathing patterns on internal target volumes in fourdimensional ct and cone-beam ct images in the context of stereotactic lung radiotherapy. Med Phys. 2013; 40:021904.

16. Rietzel E, Pan T, Chen GT. Four-dimensional computed tomography: image formation and clinical protocol. Med Phys. 2005; 32:874-89.

17. George R, Chung TD, Vedam SS, Ramakrishnan V, Mohan R, Weiss E, Keall PJ. Audio-visual biofeedback for respiratorygated radiotherapy: impact of audio instruction and audio-visual biofeedback on respiratorygated radiotherapy. Int J Radiat Oncol Biol Phys. 2006; 65: 924-933.

18. Balter JM, Lam KL, McGinn CJ, Lawrence TS, Ten Haken RK. Improvement of CT-based treatment-planning models of abdominal targets using static exhale imaging. Int J Radiat Oncol Biol Phys. 1998; 41:939-43.

19. Dinkel J, Welzel T, Bolte H, Hoffmann B, Thierfelder C, Mende U, Debus J, Heller M, Kauczor HU, Biederer J. Four-dimensional multislice helical CT of thelung: qualitative comparison of retrospectively gated and static images in anex-vivo system. Radiother Oncol. 2007; $85: 215-22$.

20. Persson GF, Nygaard DE, Munck Af Rosenschöld P, Richter Vogelius I, Josipovic M, Specht L, Korreman SS. Artifacts in conventional computed tomography (CT) and free breathing four-dimensional CT induce uncertainty in gross tumor volume determination. Int J Radiat Oncol Biol Phys. 2011; 80:1573-80.

21. Li F, Li J, Ma Z, Zhang Y, Xing J, Qi H, Shang D. Comparison of internal target volumes defined on 3-dimensional, 4-dimensonal, and cone-beam CT images of non-small-cell lung cancer. Onco Targets Ther. 2016; 9:6945-6951.

22. Ezhil M, Vedam S, Balter P, Choi B, Mirkovic D, Starkschall G, Chang JY. Determination of patient-specific internal gross tumor volumes for lung cancer using fourdimensional computed tomography. Radiat Oncol. 2009; $4: 1-14$.

23. Zhao KL, Liao Z, Bucci MK, Komaki R, Cox JD, Yu ZH, Zhang L, Mohan R, Dong L. Evaluation of respiratoryinduced target motion for esophageal tumors at the gastroesophageal junction. Radiother Oncol. 2007; 84:283-9.

24. Wang W, Li J, Zhang Y, Shao Q, Xu M, Guo B, Shang D. Correlation of primary middle and distal esophageal cancers motion with surrounding tissues using four-dimensional computed tomography. Onco Targets Ther. 2016; 9:3705-10.

25. Seppenwoolde Y, Shirato H, Kitamura K, Shimizu S, Van Herk M, Lebesque JV, Miyasaka K. Precise and real-time measurement of 3D tumor motion in lung due to breathing and heartbeat, measured during radiotherapy. Int J Radiat Oncol Biol Phys. 2002; 53:822-34.

26. Vali FS, Nagda S, Hall W, Sinacore J, Gao M, Lee SH, Hong R, Shoup M, Emami B. Comparison of standardized uptake value-based positron emission tomography and computed tomography target volumes in esophageal cancer patients undergoing radiotherapy. Int J Radiat Oncol Biol Phys. 2010; 78:1057-63.

27. Vergalasova I, Maurer J, Yin FF. Potential underestimation of the internal target volume ITV from free-breathing CBCT. Med Phys. 2011; 38:4689-99.

28. Boswell S, Tomé W, Jeraj R, Jaradat H, Mackie TR. Automatic registration of megavoltage to kilovoltage CT images in helical tomotherapy: an evaluation of the setup verification process for the special case of a rigid head phantom. Med Phys. 2006; 33:4395-404. 